\title{
Clinical Benefits of Oral Anticoagulant Use in Cancer Patients at Increased Risk for Venous Thromboembolism per Khorana Index
}

\author{
Yeo Jin Choi $\mathbb{D}^{1, *}$ \\ Yong Won $\mathrm{Choi}^{2, *}$ \\ Jung-woo Chae (D) $^{3, *}$ \\ Hwi-yeol Yun (1D ${ }^{3}$ \\ Sooyoung Shin $\mathbb{1}^{4,5}$ \\ 'Department of Clinical Pharmacy, \\ Graduate School of Clinical Pharmacy, \\ CHA University, Seongnam, Gyeonggi-do, \\ 13488, Republic of Korea; ${ }^{2}$ Department \\ of Hematology-Oncology, School of \\ Medicine, Ajou University, Suwon, \\ Gyeonggi-do, 16499, Republic of Korea; \\ ${ }^{3}$ College of Pharmacy, Chungnam \\ National University, Daejeon, 34134, \\ Republic of Korea; ${ }^{4}$ College of Pharmacy, \\ Ajou University, Suwon, Gyeonggi-do, \\ 16499, Republic of Korea; ${ }^{5}$ Research \\ Institute of Pharmaceutical Science and \\ Technology (RIPST), Ajou University, \\ Suwon, Gyeonggi-do, 16499, Republic of \\ Korea
}

*These authors contributed equally to this work
Correspondence: Sooyoung Shin;

Hwi-yeol Yun

Tel +82 31 $2193456 ;+82428215941$

Fax +82 31 $2193435 ;+82428236566$

Email syshin@ajou.ac.kr;

hyyun@cnu.ac.kr
Background: Cancer patients are at increased risk for venous thromboembolism (VTE) due to cancer-induced hypercoagulability. However, current guidelines do not routinely recommend prophylactic use of oral anticoagulants to prevent VTE in cancer patients.

Objective: To evaluate the efficacy and safety of novel oral anticoagulants (NOACs) versus no anticoagulant use (no-use) and, additionally, differential effects between NOACs and warfarin, in VTE and adverse bleeding prevention among cancer patients, in consideration of risk stratification by gender, high-risk chemotherapy exposure, and Khorana index.

Methods: This national health insurance data-based study with a 180-day follow-up enrolled cancer patients with or without oral anticoagulant use in 2017. The primary outcome was VTE risk in oral anticoagulant users vs non-users. Four propensity score-matched comparison pairs were designed: use vs no-use, NOAC vs no-use, warfarin vs no-use, and NOAC vs warfarin. A logistic regression model was used to investigate between-group differences in VTE and bleeding risk.

Results: When compared to no-use, NOACs showed substantial effects in preventing VTE complications $(\mathrm{OR}=0.40, p<0.001)$, primarily deep vein thrombosis (DVT) events $(\mathrm{OR}=0.38$, $p<0.001$ ), in both male and female cancer patients as well as those with a Khorana score $\geq 1$. Adverse bleeding risk was comparable or lower in NOAC-receiving female patients $(p=0.13)$ and male patients $(p=0.04)$, respectively. In contrast, no protective effects were found with warfarin compared to no-use in controlling thrombosis and adverse bleeding risk. In a headto-head comparison of NOACs versus warfarin, DVT risk in those patients exposed to highrisk chemotherapy was significantly decreased with NOAC use (OR=0.19, $p=0.03)$.

Conclusion: NOACs can be a promising thromboprophylactic option in both male and female cancer patients with VTE risk.

Keywords: venous thromboembolism, cancer, oral anticoagulant, Khorana

\section{Introduction}

Cancer patients are at increased risk of developing venous thromboembolism (VTE) complications involving deep vein thrombosis (DVT) and pulmonary embolism (PE) due to cancer-induced hypercoagulability, complicated by repeated chemotherapy administration through central or peripheral venous catheters, vascular endothelial damage, blood flow obstruction by tumor masses, procoagulant microparticles from cancer cells, comorbid conditions, advanced age, and impaired mobility. ${ }^{1-4}$ VTE has a substantial impact on cancer patients as it requires inpatient treatment and rehabilitation, consequently increasing health-care costs and 
undermining patients' quality of life. ${ }^{3-6}$ However, current guidelines do not routinely recommend the prophylactic use of oral anticoagulant therapy to prevent VTE events in ambulatory cancer patients; the guidelines recommend therapeutic anticoagulation for the treatment of VTE encountered during the course of cancer management. ${ }^{7,8}$

VTE treatment typically requires parenteral anticoagulation at therapeutic doses, primarily based on lowmolecular weight heparin (LMWH) or unfractionated heparin (UFH). ${ }^{7,8}$ However, parenteral anticoagulation requires inpatient care as doses need to be given multiple times daily or by continuous infusion; ${ }^{7}$ besides, the known risk of major bleeding and high cost also negatively affects patient adherence and subsequently patient outcomes. ${ }^{9}$ Cancer patients are susceptible to major bleeding secondary to multiple comorbidities, myelosuppression induced by cancer-directed therapies and complexed interaction potentials with concomitant medications that can also increase bleeding risk; ${ }^{10-12}$ hence, parenteral anticoagulation may further exacerbate the risk of major bleeding in these predisposed patients. There have been only a few randomized controlled trials (RCTs) conducted thus far that investigated the potential of oral anticoagulants for thromboprophylaxis in cancer patients. ${ }^{13,14}$ A recent RCT compared the efficacy of apixaban versus placebo over a follow-up period of 180 days and reported that apixaban can be an effective and safe thromboprophylactic option in cancer patients. ${ }^{13}$ In another RCT, rivaroxaban use, when compared against placebo, led to a significantly decreased incidence of VTE as well as major bleeding during the 180-day follow-up in high-risk ambulatory patients with cancer. ${ }^{14}$ Oral anticoagulants possess advantages over parenteral agents as thromboprophylaxis in that they are less likely to cause major bleeding and more practical to be used by ambulatory patients..$^{9,15}$ Hence, there is a pressing need to evaluate the efficacy and safety of oral anticoagulant-based thromboprophylaxis in cancer patients at an increased risk for VTE complications, thereby improving clinical outcomes and prognosis of these patient populations.

The Khorana risk score is a validated stratification scoring system used for early identification and intervention for cancer patients at risk for VTE development, but the clinical relevance of this scoring system in real-world practice settings has yet to be fully established. ${ }^{2,16}$ In addition, female patients and those exposed to high-risk chemotherapy are known to be more prone to blood clots than other cancer patients, ${ }^{1,17}$ but studies evaluating these factors in clinical outcomes of oral anticoagulation among cancer patients are still lacking. Therefore, in this retrospective cohort study, we aimed to evaluate the efficacy and safety of novel oral anticoagulants (NOACs) versus no anticoagulation (no-use) and, additionally, potential differential effects between NOACs and warfarin, in the prevention of VTE and adverse bleeding events among cancer patients, in consideration of risk stratification by patient gender, high-risk chemotherapy exposure, and Khorana index.

\section{Methods}

\section{Study Population}

A retrospective cohort study involving cancer patients, with or without oral anticoagulant use, was performed using the Korean Health Insurance Review \& Assessment Service-National Patients Sample-2017 (HIRA-NPS-2017-0031). The HIRA database contains administrative National Health Insurance (NHI) claims data related to health-care services provided for the entire national beneficiaries, which include patient demographics, diagnoses per the International Classification of Disease 10th Revision (ICD-10), procedures, and comprehensive medical utilization, along with prescription data. The initial samples of 1,473,083 patients or three percent of the entire beneficiaries in 2017 were collected using a stratified randomized sampling method to ensure national representativeness. The present study patients were then identified if patients had a hospital encounter associated with a cancer diagnosis per ICD-10 code more than once in 2017. Prespecified exclusion criteria included prior dialysis treatment, preexisting end-stage renal disease, renal transplant status at study entry, and active VTE requiring inpatient treatment with parenteral anticoagulation. The study protocol was approved by the Institutional Review Board of Ajou University (202004-HB-EX-001). Due to the retrospective design of the study based on anonymized health insurance data, informed consent from study participants was waived. No further ethics approval was required as the HIRA authorized the researchers to analyze de-identified nationwide patient data for research purposes.

\section{Study Medications and Variables}

Cancer patients who were treated with oral anticoagulation for $\geq 30$ days were categorized as anticoagulant users and those without such medication as non-users. Anticoagulant 
users were then classified into two cohorts of either warfarin or NOAC users, in accordance with their primary anticoagulant therapy that lasted for $\geq 30$ consecutive days; NOAC agents include rivaroxaban, apixaban, dabigatran, and edoxaban. Those patients with a history of both treatments for an equivalent length of time, which was defined as the treatment duration not differing by $\geq 50 \%$ of each other's prescribed days, were excluded from further analysis, ${ }^{18}$ leading to two mutually exclusive user groups. Predetermined variables included patient demographics, such as age and gender, cancer sites, Khorana scores, comorbidities, Charlson comorbidity index (CCI) scores, exposure to chemotherapy with high thrombotic risk, including tamoxifen, thalidomide, lenalidomide, cisplatin, fluorouracil, L-asparaginase, bevacizumab, ${ }^{17}$ along with concomitant medications with bleeding risk, such as antiplatelets, nonsteroidal anti-inflammatory drugs (NSAIDs), corticosteroids, and parenteral anticoagulants. All eligible prescription data for comedication assessment were captured using a total of 107 relevant substance codes listed in the national formulary, which belong to the aforementioned drug categories. VTE risk of individual patients was estimated by using the Khorana index system, which takes into account cancer sites, hematologic status in terms of hemoglobin, platelet and leukocyte levels, and obesity status. ${ }^{16}$ The following clinically relevant comorbid conditions were identified per ICD-10 code: renal disease, diabetes without complications, diabetes with complications, hemorrhagic stroke, ischemic stroke, cerebrovascular occlusion, and atrial fibrillation, and previous VTE.

\section{Study Outcomes}

The primary outcome was the incidence and risk of VTE in cancer patients receiving oral anticoagulants versus those not receiving such medication, stratified by gender, high-risk chemotherapy exposure, and Khorana index to account for differential effects of oral anticoagulation per different patient attributes. The VTE endpoint was a composite of newly diagnosed DVT or PE identified via health-care visit episodes for the respective events that occurred up to 180 days post the index date; betweengroup differences in VTE risk were then evaluated by computing odds ratios (ORs) along with 95\% confidence intervals (CIs). The index date for study participants was defined as the first encounter date associated with a cancer diagnosis in each patient. To better assess differential effects of NOACs versus warfarin on VTE complications in cancer patients, a separate risk analysis was designed, where NOACs were collectively compared against warfarin. The safety outcome was a composite of hospital encounter with a primary diagnosis of bleeding, such as cerebrovascular hemorrhage, hemorrhage of esophagus, hematemesis, melena, gastrointestinal hemorrhage, hemorrhage from respiratory passages, hemorrhage of anus and rectum, and hemorrhage not elsewhere classified. Patient follow-up began on the index date until the earliest occurrence of any of the censoring events as follows: study endpoint events, follow-up discontinuation before the end of study period, or the end of the 180-day follow-up. The outcome date was determined as the earliest date when a patient encountered a given endpoint event during the study period, but to enhance the quality and reliability of this retrospective health insurance data-based study, only those cases that occurred at least 7 days after the index date were captured as a relevant event and incorporated in risk analyses.

\section{Statistical Analysis}

We designed four comparison pairs: use versus no-use, NOAC versus no-use, warfarin versus no-use, and lastly a head-to-head comparison of NOAC versus warfarin. To balance potential confounding factors between different study cohorts, eligible patients were matched in a $1: 1$ ratio to each of the two comparison groups, based on propensity score (PS) matching per relevant patient characteristics, in terms of age category, gender, previous VTE and comorbidity at study entry, high-risk chemotherapy exposure and comedication patterns during the follow-up. The multinomial PS for individual patients was estimated by fitting a logistic regression model accounting for the aforementioned pre-treatment variables as covariates. A separate 1:1 PS-matching process was repeated for each of the four comparison pairs; the caliper matching method was utilized to improve the quality of matching due to heterogeneity with respect to baseline comorbidities and comedication patterns between initial cohorts. The multiple logistic regression analysis was also employed to adjust for potential confounding factors in PS-matched study patients. We calculated the incidence rates of endpoint events as well as ORs along with $95 \%$ CIs by comparing the user group as a whole and then the NOAC and warfarin groups separately against the non-user group (reference), respectively; then a head-to-head comparison between NOAC and warfarin (reference) was additionally performed. Risk analyses were first conducted for incident endpoint 
events regardless of patient factors relevant for VTE risk assessment, and then repeated in each stratum by gender, high-risk chemotherapy exposure, and Khorana index at study entry. The $p$-values were two-sided and deemed statistically significant if $<0.05$. Statistical analyses were performed using SAS 9.4 software (SAS Institute Inc., Cary, NC, USA).

\section{Results}

\section{Characteristics of Study Patients}

Of the 1,473,083 sampled patients, adult patients with cancer, including both oral anticoagulant users and non-users, were screened, and resultantly 47,454 patients were found eligible for study entry. A total of 238 patients were then excluded from further analysis due to the following reasons: previous dialysis treatment or end-stage renal disease $(n=167)$, kidney transplant status at study entry $(\mathrm{n}=44)$, and not being adult $(n=27)$; no patients were further excluded due to the equivalent duration of study therapies or active VTEs associated with parenteral anticoagulant treatment. Of the remaining 47,216 patients (636 in the NOAC cohort, 298 in the warfarin cohort, and 46,282 in the no-use cohort), 1:1 PS matching was performed in each of the four comparison pairs. As a result, 870 patients in each of the use and no-use groups, 601 patients in each of the NOAC and no-use groups, 298 patients in each of the warfarin and no-use groups, and 298 patients in the NOAC and warfarin groups were identified for study analysis, respectively. Baseline characteristics of the PS-matched study patients per individual comparison pairs are summarized in Table 1. Overall, the distribution of gender, common cancer sites, Khorana index, comorbidity burden in terms of diabetes with or without complications and hemorrhagic stroke, was well balanced between comparison groups post PS matching. Khorana scores suggested equivalent VTE risk at study entry across study cohorts. As summarized in Table S1, baseline attributes of the initial cohort of 47,216 cancer patients prior to PS matching showed an inherent disparity between oral anticoagulant users versus non-users, in terms of baseline comorbidities and comedication patterns; we further adjusted for potential confounding factors in PS-matched study patients by employing the multiple logistic regression analysis.

\section{Study Outcomes: Use versus No-Use}

The incidence and risk of VTE events in oral anticoagulant users as compared to non-users were assessed in the PSmatched groups and the results are presented in Figure 1.
Female patients were more susceptible to thrombosis complications, both DVT and PE, than male patients; the gender difference was greatest in DVT rates: $10.5 \%$ versus $5.3 \%$ in females and males, respectively, nearly a twofold difference $(p<0.001)$. The primary outcome of VTE was encountered in $86(9.9 \%)$ and $111(12.8 \%)$ patients in users and non-users, respectively. The ORs were adjusted for potential confounding factors as indicated in the distribution of patient characteristics at baseline. Anticoagulant use in cancer patients had a significant protective effect in preventing VTE complications compared to non-users: the OR $(95 \% \mathrm{CI})$ was 0.52 $(0.38-0.71)$ in overall patients, $0.50(0.32-0.78)$ in male patients, $0.53(0.35-0.82)$ in female patients, $0.47(0.33-0.66)$ in those not exposed to high-risk chemotherapy, and 0.51 $(0.37-0.71)$ and $0.57(0.37-0.89)$ in those with a Khorana score of $\geq 1$ and $\geq 2$, respectively. A lower risk of DVT complications was detected among anticoagulant users compared to non-users and the protective effect remained significant in all strata: the OR $(95 \% \mathrm{CI})$ was $0.51(0.35-0.74)$ in overall patients, $0.54(0.31-0.96)$ in males, $0.48(0.29-0.80)$ in females, $0.43(0.29-0.66)$ in those with no exposure to highrisk chemotherapy, and $0.51(0.34-0.76)$ in those with a Khorana score of $\geq 1$. Such efficacy was not found in the prevention of PE events in overall and female cancer patients and in all strata by high-risk chemotherapy exposure and Khorana index, except in male patients where potential protective effects were detected with oral anticoagulant use: the incidence of PE was 3.5\% versus $4.4 \%$ in the user and nonuser groups, respectively ( $p=0.046$ ). Interestingly, oral anticoagulant users were assessed less likely to experience bleeding events than non-users. Here, the ORs have been adjusted for relevant baseline factors, especially concomitant antiplatelet, NSAID, and corticosteroid use, all with potential bleeding risk; use rates of those medications tended to be higher among anticoagulant non-users. More detailed results are provided in Table S2.

\section{Study Outcomes: NOAC versus No-Use}

Figure 2 visually summarizes the results of incidence rates and comparative risk of primary endpoints of VTE complications among the PS-matched study patients of NOAC users versus non-users; the ORs associated with each component of VTE composite endpoint, DVT and PE, were also separately presented. Similar patterns of gender differences in thrombosis rates were observed; female patients were more prone to develop VTE compared to male patients $(16.7 \%$ versus $10.2 \%, p<0.001)$, both DVT $(11.2 \%$ versus $5.9 \%$, $p=0.001)$ and PE $(7.2 \%$ versus $4.9 \%, p=0.11)$. The ORs 


\begin{tabular}{|c|c|c|c|c|c|c|c|}
\hline$\frac{0}{\frac{0}{\pi N}}$ & $\stackrel{m}{0}$ & 市 & ợ & $\frac{0}{0}$ & ồ & 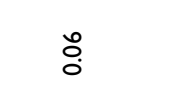 & 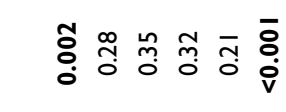 \\
\hline 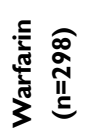 & 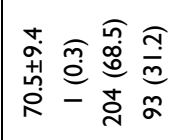 & $\begin{array}{l}\widehat{\sigma} \\
\stackrel{\rho}{\rho} \\
\underline{\infty}\end{array}$ & 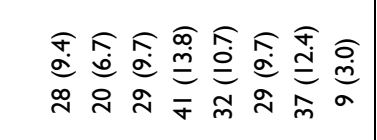 & 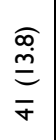 & 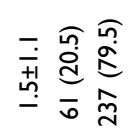 & 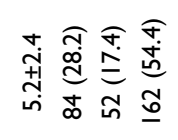 & 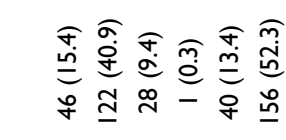 \\
\hline 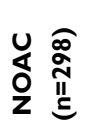 & 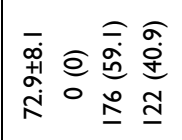 & 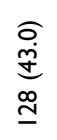 & 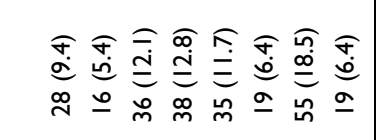 & 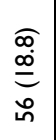 & 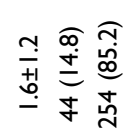 & 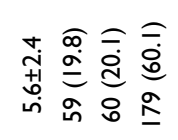 & 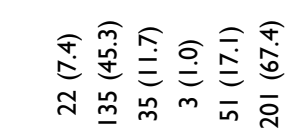 \\
\hline 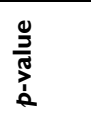 & $\stackrel{\text { do }}{0}$ & $\stackrel{\circ}{\circ}$ & î̀. & 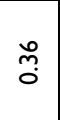 & $\stackrel{2}{\circ}$ & 吕 & 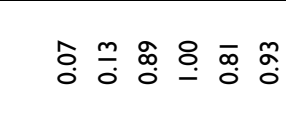 \\
\hline 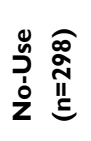 & 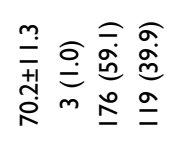 & 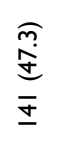 & 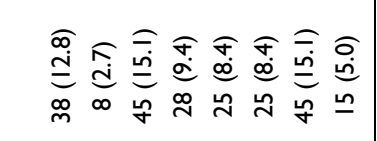 & $\begin{array}{l}\widehat{f} \\
\stackrel{d}{=} \\
o \\
o\end{array}$ & 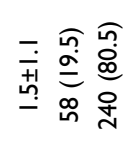 & 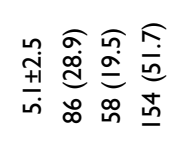 & 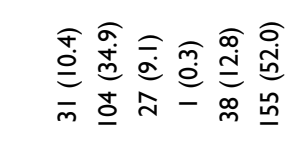 \\
\hline 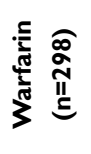 & 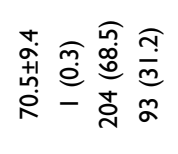 & $\begin{array}{l}\widehat{\sigma} \\
\stackrel{0}{\rho} \\
\underline{\infty}\end{array}$ & 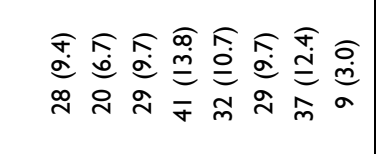 & 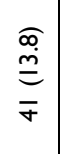 & 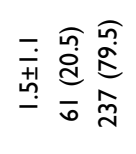 & 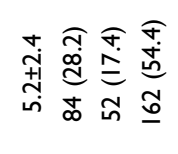 & 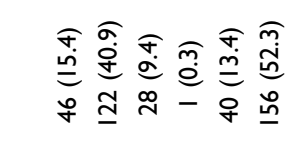 \\
\hline 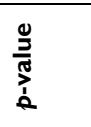 & $\stackrel{\infty}{\widetilde{o}}$ & 范 & 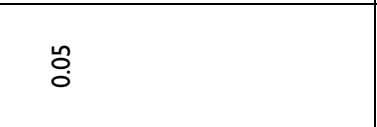 & $\stackrel{\circ}{\circ}$ & $\stackrel{0}{\circ}$ & ֻัণ & 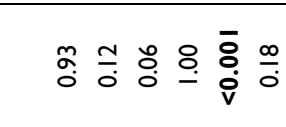 \\
\hline 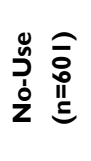 & 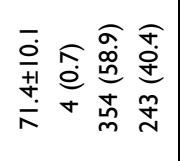 & $\begin{array}{l}\widehat{\infty} \\
\infty \\
\stackrel{\infty}{\infty} \\
\stackrel{\sim}{\sim}\end{array}$ & 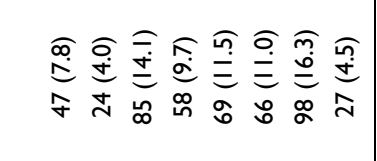 & 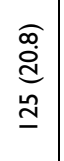 & 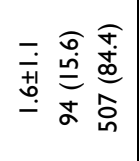 & 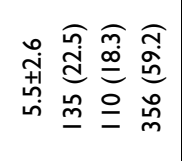 & 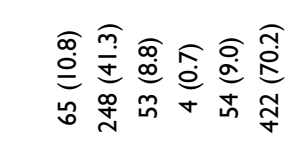 \\
\hline 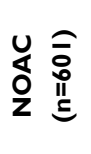 & 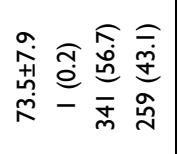 & $\begin{array}{l}\widehat{\overline{\dot{d}}} \\
\bar{\Phi}\end{array}$ & 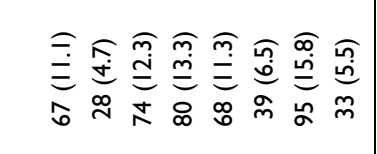 & $\begin{array}{l}\widehat{o} \\
\stackrel{\stackrel{0}{0}}{\circ} \\
\underline{8}\end{array}$ & 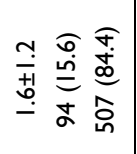 & 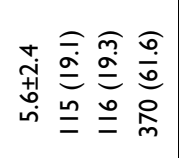 & 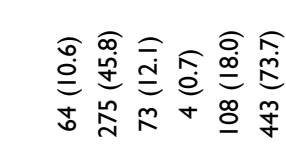 \\
\hline 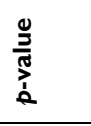 & o̊. & $\begin{array}{l}\stackrel{L}{\infty} \\
\infty \\
0\end{array}$ & స్ & $\hat{0}$ & ờ & ઼ָ & 응 官 \\
\hline 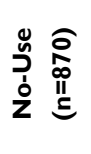 & 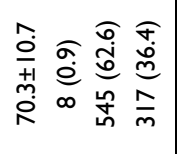 & 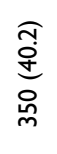 & 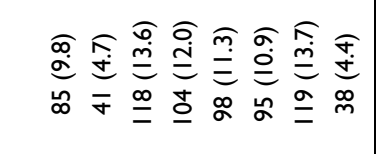 & $\begin{array}{l}\underset{0}{\infty} \\
\stackrel{\infty}{\stackrel{\infty}{\infty}} \\
\underline{\underline{w}}\end{array}$ & 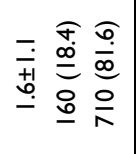 & 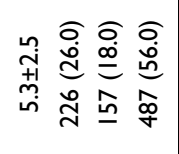 & 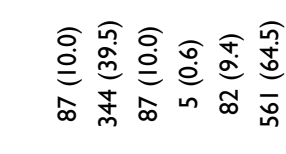 \\
\hline : & 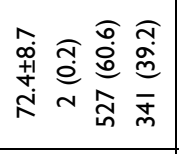 & 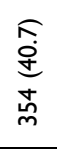 & 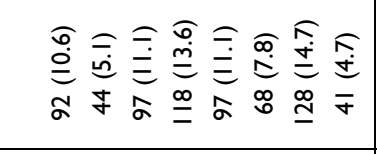 & 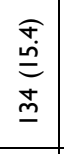 & 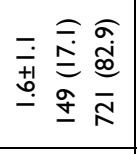 & 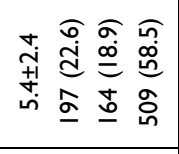 & 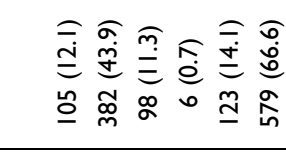 \\
\hline 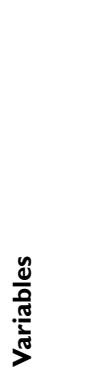 & 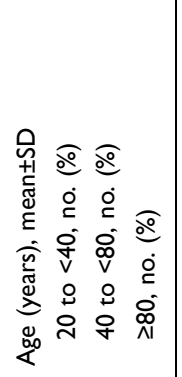 & 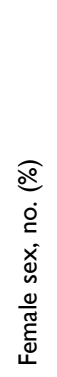 & 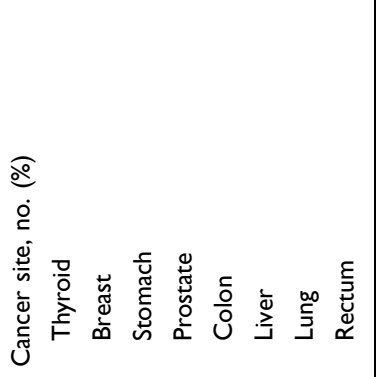 & 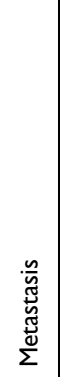 & 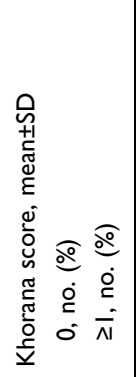 & 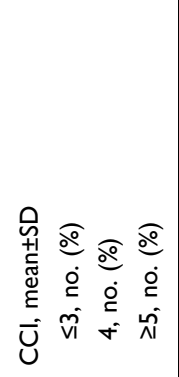 & 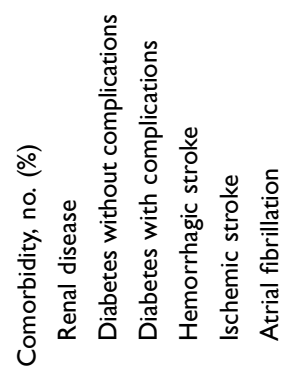 \\
\hline
\end{tabular}




\begin{tabular}{|c|c|c|c|}
\hline$\frac{9}{\frac{D}{\pi}}$ & $\underset{0}{0}$ & 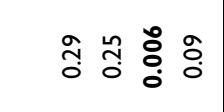 & $\begin{array}{l}\bar{o} \\
\dot{0} \\
\mathrm{v}\end{array}$ \\
\hline 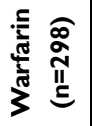 & $\frac{\widehat{\bar{\infty}}}{\stackrel{ \pm}{\sim}}$ & 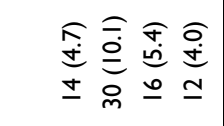 & 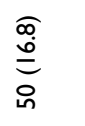 \\
\hline 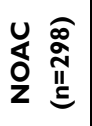 & 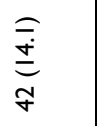 & 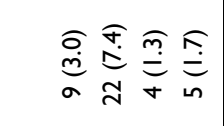 & $\begin{array}{l}\underset{\sigma}{\infty} \\
\stackrel{d}{d} \\
\stackrel{\circ}{\infty}\end{array}$ \\
\hline 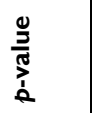 & 苞 & 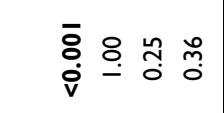 & ō \\
\hline 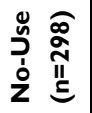 & 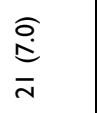 & 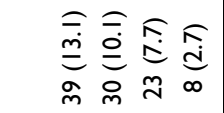 & $\underset{\bar{N}}{\stackrel{\bar{N}}{ \pm}}$ \\
\hline 而 & $\widehat{\widehat{\bar{\infty}}}$ & 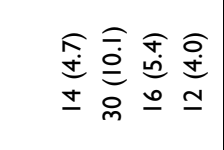 & 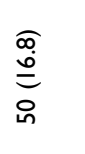 \\
\hline$\frac{0}{\frac{D}{\pi j}}$ & 饣ñ & 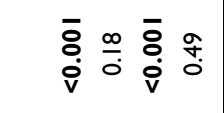 & $\frac{n}{0}$ \\
\hline 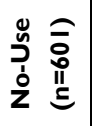 & 晜 & 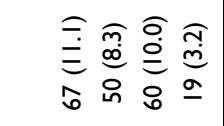 & 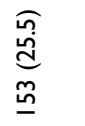 \\
\hline 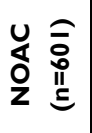 & 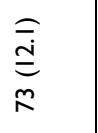 & 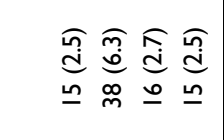 & $\begin{array}{l}\underset{\mathfrak{d}}{d} \\
\underline{\underline{m}}\end{array}$ \\
\hline$\frac{\mathscr{g}}{\pi}$ & 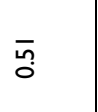 & 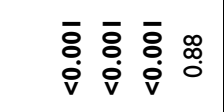 & $\stackrel{\infty}{\infty}$ \\
\hline 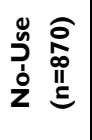 & $\underset{\substack{\circ \\
\stackrel{0}{\circ}}}{\stackrel{0}{\wedge}}$ & 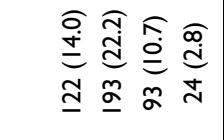 & $\begin{array}{l}\widehat{\infty} \\
\stackrel{d}{0} \\
\underline{\infty}\end{array}$ \\
\hline م. & $\begin{array}{l}\hat{\sigma} \\
\stackrel{\sigma}{\infty} \\
\stackrel{\infty}{\infty}\end{array}$ & 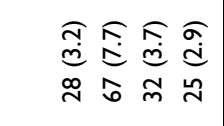 & $\begin{array}{l}\widehat{n} \\
\stackrel{0}{0} \\
\infty \\
\underline{\Sigma}\end{array}$ \\
\hline 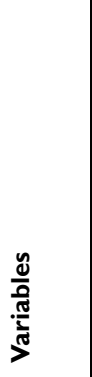 & 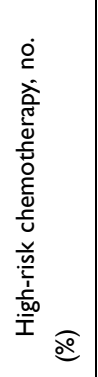 & 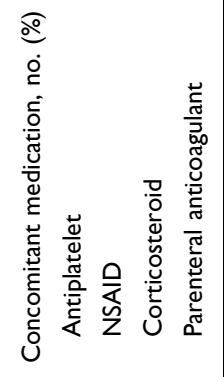 & 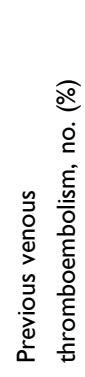 \\
\hline
\end{tabular}

were adjusted for potential confounding factors. Cancer patients on NOAC therapy were at substantially reduced risk for VTE development relative to non-users: the OR (95\% CI) was $0.40(0.28-0.58)$ in overall patients. The beneficial effects remained statistically significant in most strata by gender and other risk factors: the OR $(95 \% \mathrm{CI})$ was $0.45(0.27-0.75)$ in males, $0.34(0.20-0.58)$ in females, 0.35 $(0.23-0.53)$ in those without exposure to high-risk chemotherapy, and $0.40(0.27-0.59)$ and $0.49(0.29-0.80)$ in those with a Khorana score of $\geq 1$ and $\geq 2$, respectively. NOACs were effective in lowering VTE risk primarily by preventing DVT events in both male and female cancer patients and also in those with no exposure to high-risk chemotherapy and with a Khorana score of $\geq 1$, as compared to no-use. NOACs were not associated with lower risk of PE with statistical significance relative to no-use in most strata, but potential protective effects were assessed in those with no exposure to chemotherapy. Notably, NOAC users, especially male patients, were less likely to have a hospital encounter for bleeding events compared with non-users; the ORs here were adjusted for the aforementioned pretreatment factors, including comedication patterns that may influence bleeding risk. More detailed results are available in Table S3.

\section{Study Outcomes: Warfarin versus No-Use}

The incidence and risk of the composite endpoint of VTE, DVT and PE components, depending on warfarin use or no-use, were examined in the overall PS-matched study patients as well as separately in each stratum by gender and VTE risk per high-risk chemotherapy exposure and Khorana index (Figure 3). The incidence of thrombosis outcome rates were higher in female patients than in male patients; the DVT rates showed a 2.6-fold difference between males and females $(11.6 \%$ versus $4.5 \%$, $p=0.001)$. There were only 298 warfarin users $(0.6 \%$ of the initial cohort of 47,216 cancer patients prior to PSmatching), who met the inclusion criteria for study entry. As there were a relatively small number of patients in the initial warfarin cohort, 298 patients not on oral anticoagulant therapy were identified by performing PS-matching for the no-use group. Most pretreatment variables were well balanced between the PS-matched groups, but potential confounders (age, antiplatelet use) were further adjusted for via subsequent multiple logistic regression analyses. The ORs indicated no beneficial effects with warfarin as compared to no-use in terms of the prevention 


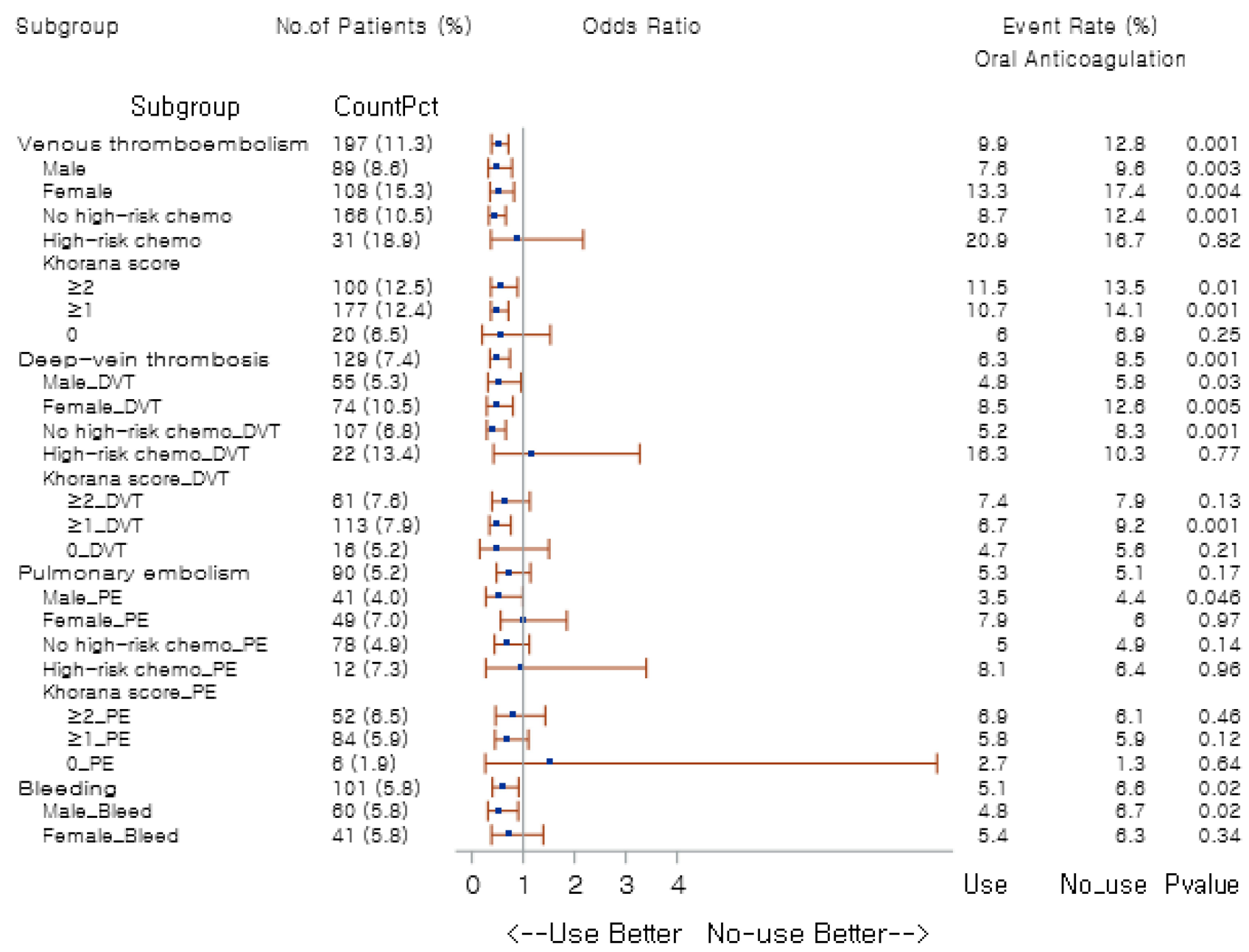

Figure I Forest plot of odds ratios by patient subgroups: use versus no-use.

Abbreviations: DVT, deep vein thrombosis; PE, pulmonary embolism.

of VTE, DVT, and PE; warfarin was not associated with increased bleeding risk. More detailed results are available in Table S4.

\section{Study Outcomes: NOAC versus Warfarin}

An additional analysis was designed for the head-to-head comparison between NOACs and warfarin (Figure 4). The PS-matched 298 cancer patients on NOACs were compared against those 298 warfarin users in terms of overall VTE risk, DVT and PE risk separately, and adverse bleeding risk. The ORs were adjusted for potential confounding variables per baseline characteristics of PS-matched study patients. Most outcomes were not associated with statistical significance except DVT risk in the high-risk chemotherapy stratum; high-risk chemotherapy-exposed cancer patients receiving NOACs were at significantly lower risk of experiencing DVT events than those on warfarin $(\mathrm{OR}=0.19, p=0.03)$. More detailed results are available in Table S5.

\section{Discussion}

In this cohort study, we evaluated the efficacy and safety of oral anticoagulants in the prevention of VTE among cancer patients. To the best of our knowledge, this is the first study that conducted risk analyses, stratified by patient gender and VTE risk per high-risk chemotherapy exposure and Khorana index, comparing oral anticoagulant use versus no-use, along with the head-to-head comparison between NOACs and warfarin among cancer patients. NOACs substantially decreased VTE complications, especially DVT events, in cancer patients as compared to no-use; the beneficial effects on thrombosis prevention were detected in both males and females, those not exposed to high-risk chemotherapy, and in those with a Khorana score $\geq 1$. Adverse bleeding risk 


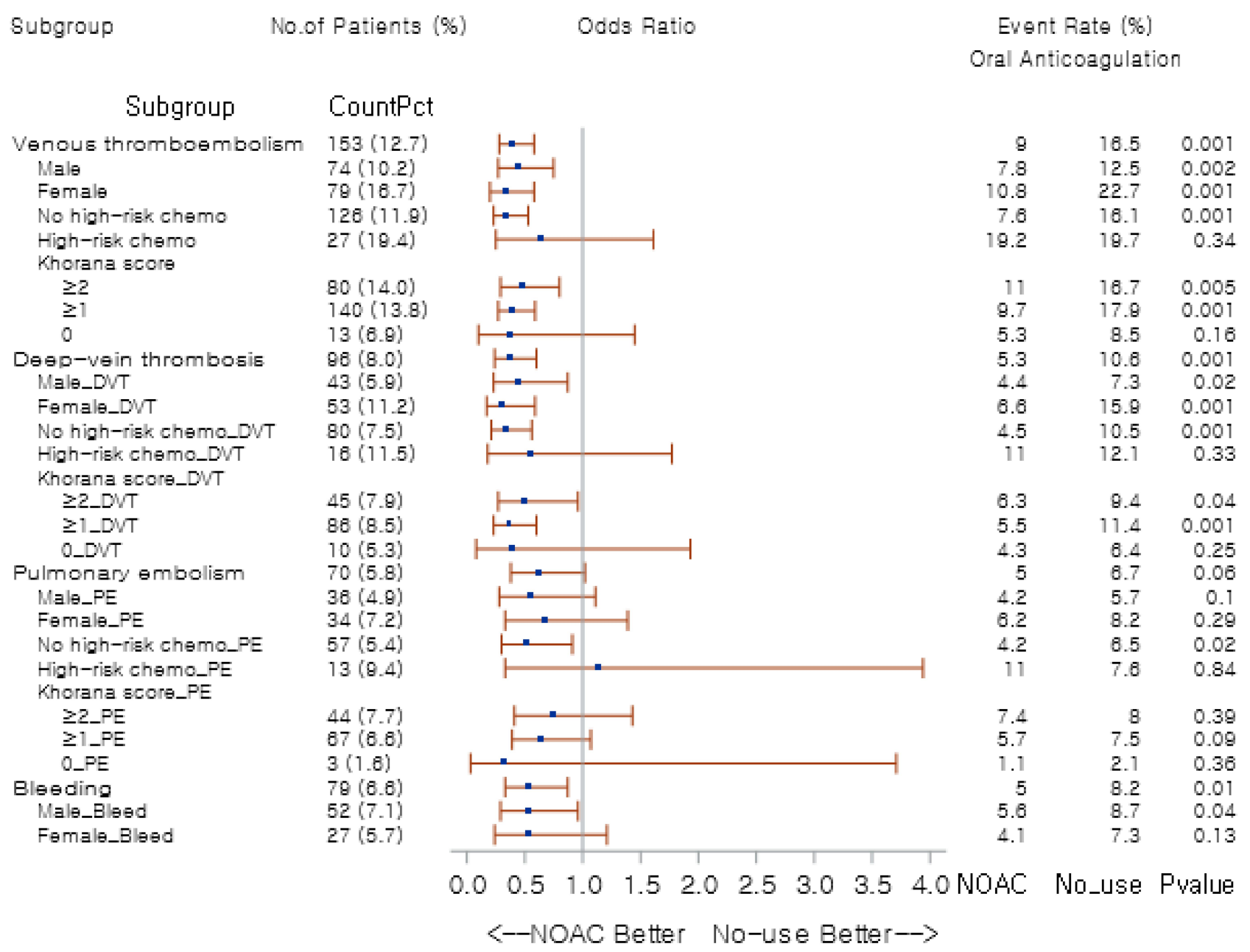

Figure 2 Forest plot of odds ratios by patient subgroups: NOAC versus no-use.

Abbreviations: DVT, deep vein thrombosis; PE, pulmonary embolism; NOAC, novel oral anticoagulant.

also appeared lower among oral anticoagulant users, especially in male patients, potentially influenced by different comedication patterns between users and non-users; nonusers showed a greater tendency to receive more concomitant medications with bleeding risk, such as antiplatelets, NSAIDs, and corticosteroids whereas oral anticoagulant users tended not to be prescribed those medications concomitantly. In contrast, no protective effects were observed with warfarin use relative to no-use in reducing the risk of thrombosis and adverse bleeding. When NOACs were compared directly against warfarin, the risk of DVT in those patients exposed to high-risk chemotherapy was substantially decreased with NOACs.

The Khorana risk score is a risk stratification tool to identify cancer patients with high risk for thrombosis complications. This tool assigns risk scores based on the following criteria: 1) cancer sites; 2) hematologic abnormalities; and 3) obesity. ${ }^{2,16}$ Any patients with a Khorana risk score of $\geq 3$ are considered as high-risk patients: ${ }^{16}$ a metaanalysis revealed the highest VTE incidence of $11 \%$ in high-risk patients while its incidence in those with lowrisk ( 0 point) and intermediate-risk (1-2 points) was $5.0 \%$ and $6.6 \%$, respectively. ${ }^{16,19}$ In our study, about $82.6 \%$ of the PS-matched study patients had a Khorana score of 1 or above (intermediate- to high-risk) ${ }^{16}$ and these patients obtained substantial benefits with oral anticoagulant use, especially with NOACs, compared to no-use in terms of the prevention of VTE complications, primarily DVT events.

Cancer patients possess at least five- to seven-fold elevated risk of VTE and 12.3 in every 1000 cancer patients develop VTE within the first six-month post cancer diagnosis. ${ }^{20,21}$ A study by Blom et al demonstrated that the risk of thrombosis was highest in the first three months of the cancer diagnosis and slowly decreased thereafter. ${ }^{3}$ Although cancer itself increases morbidity and mortality 


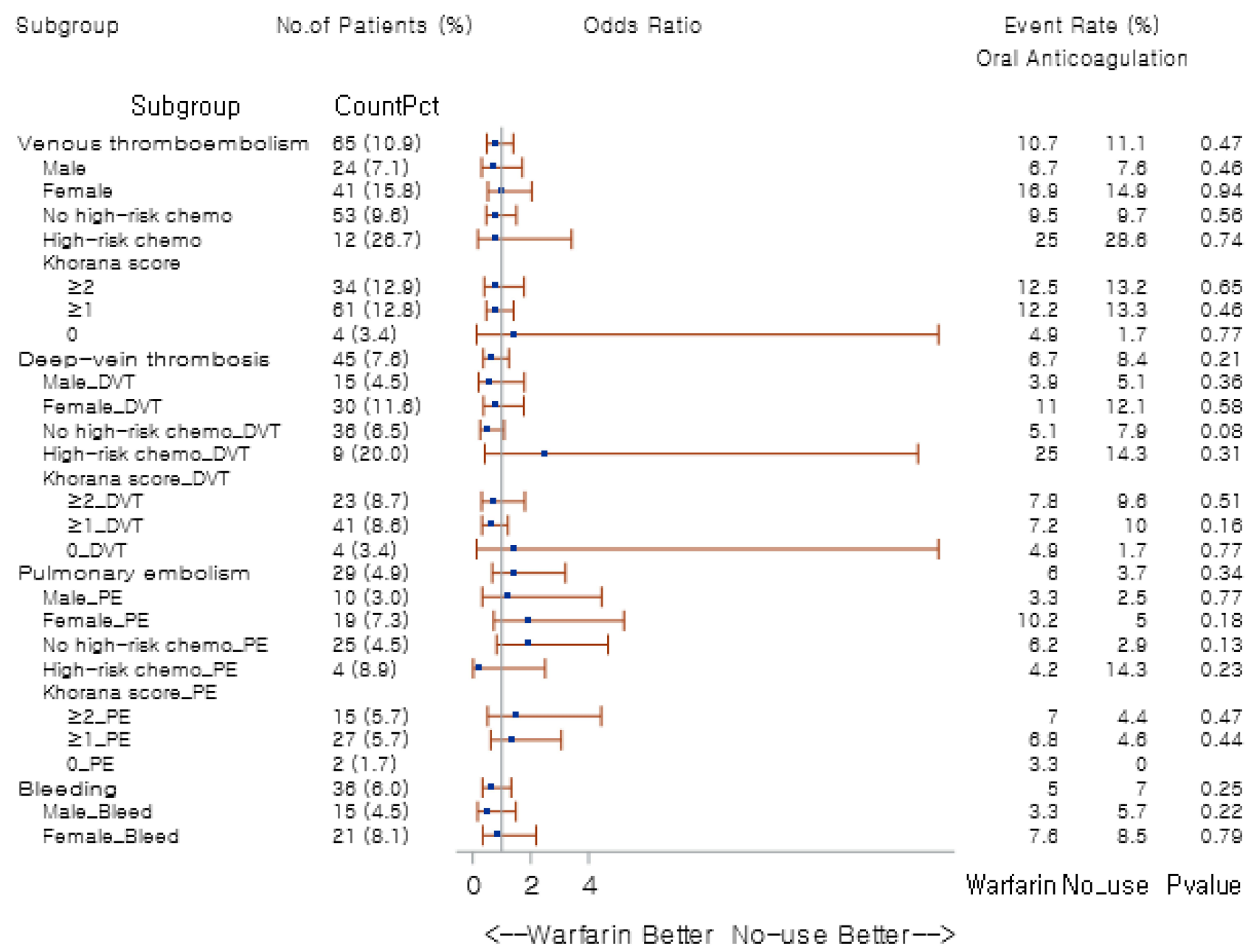

Figure 3 Forest plot of odds ratios by patient subgroups: warfarin versus no-use. Abbreviations: DVT, deep vein thrombosis; PE, pulmonary embolism.

of the patients, VTE development within the first year of the cancer diagnosis substantially worsens patient prognosis as well as cancer-related complications. ${ }^{6}$ The exact molecular mechanism and etiologies of hypercoagulable states in cancer patients have not been fully elucidated. However, numerous studies have identified the risk factors associated with prothrombotic states, and cancer itself, depending on its type, stage and grade (along with use of intravenous access devices, blood flow obstruction by tumor masses, vascular endothelial damage, procoagulant microparticles from cancer cells) has been reported as one of the strong risk factors for the hematologic dysfunction. ${ }^{1-4,6}$

The risk of cancer-associated VTE varies across different sites of cancer, as implied by the Khorana risk scoring system; ${ }^{16}$ those diagnosed with lung, lymphoma, gynecologic, bladder or testicular cancers are classified as high risk patients for VTE, and the risk is greater, by threefold or more, in those with gastric or pancreatic cancers. ${ }^{16}$ The stage of cancer is another important risk factor for cancerassociated VTE. ${ }^{5}$ According to a population-based study, cancer patients had a fourfold higher risk of VTE in the initial stage as compared to healthy counterparts, and the risk increased 58-fold in solid tumor patients with distant metastasis. ${ }^{3}$ Among various factors involved in coagulation, tissue factor (TF), a triggering factor for the extrinsic coagulation cascade, is considered as one of the most contributing components to cancer-associated prothrombotic pathways. ${ }^{6,22,23} \mathrm{TF}$ is a transmembrane protein responsible for activating the extrinsic coagulation pathway as well as platelets, via proteolysis of factor VII (FVII) to its activated form (FVIIa) and via thrombin generation, respectively. ${ }^{6,22,23} \mathrm{TF}$ is typically overexpressed in tumor cells of the aforementioned high-risk cancer sites. ${ }^{16}$ Moreover, TF expression has a strong correlation with the tumor progression secondary to TF-mediated tumor 


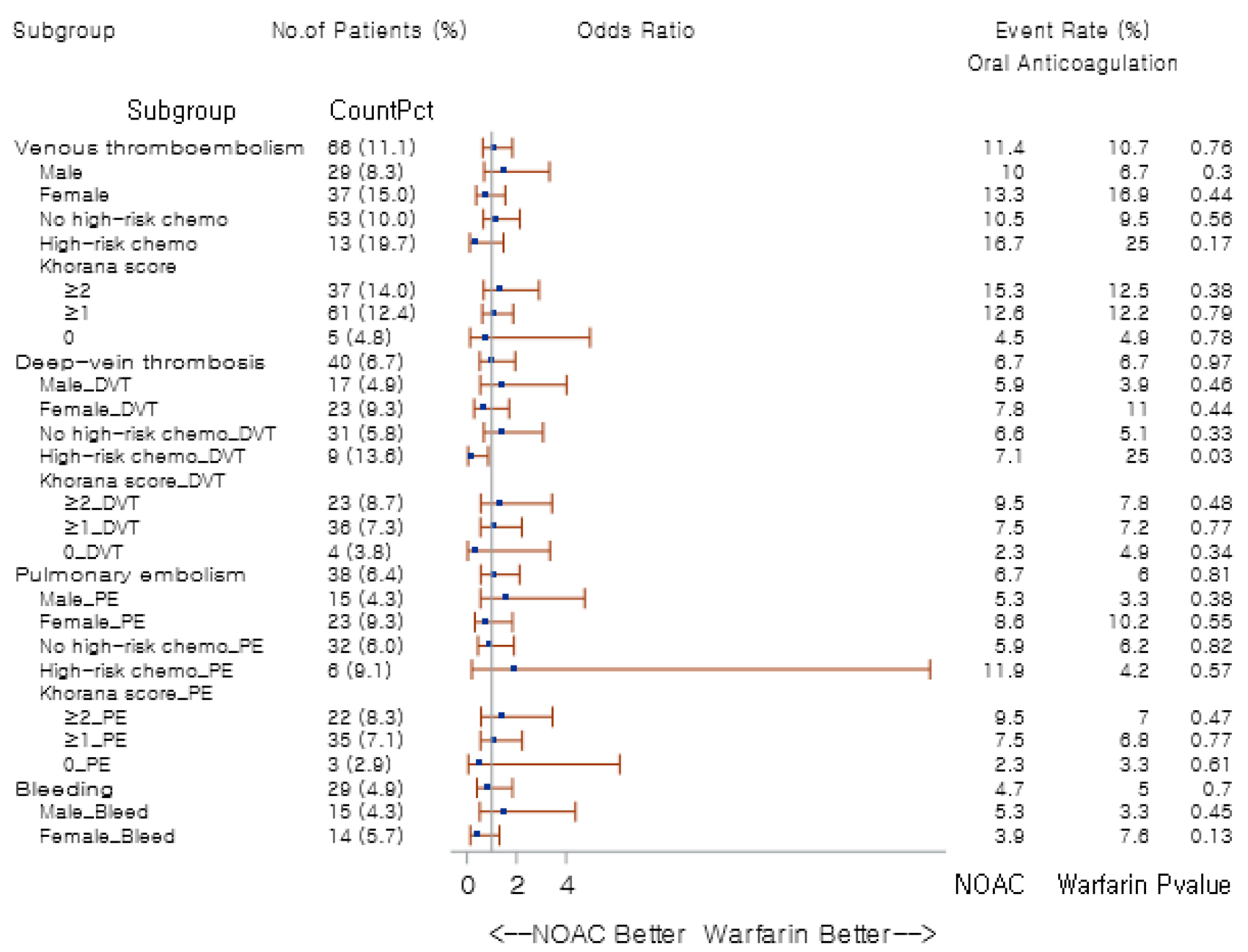

Figure 4 Forest plot of odds ratios by patient subgroups: NOAC versus warfarin.

Abbreviations: DVT, deep vein thrombosis; PE, pulmonary embolism; NOAC, novel oral anticoagulant.

angiogenesis and metastasis, which further aggravates VTE risk in those patients with advanced-stage cancers. ${ }^{24}$ Recent observations have reported the presence of contact phase activation in breast, lung, gastrointestinal, and prostate cancers, which has also been suggested as a mechanism of cancer-associated hypercoagulability. ${ }^{4}$

In addition, cancer patients have patient-specific risk factors for VTE complications, such as diverse tumor marker, old age, repeated stressor events (chemotherapy or surgery), frailty and impaired mobility. ${ }^{17,25}$ Chemotherapy increased VTE risks by six to seven folds in these patients. ${ }^{2,26}$ Based on the results from a 25-year population study, the VTE incidence increased exponentially with age, and in the context of cancer incidence typically rising with age, the risk of VTE in cancer patients could be more amplified than reported in studies. ${ }^{27,28}$ Furthermore, cancer patients have multiple underlying comorbid conditions that could also lead to an elevated VTE risk, including hypertension, hyperlipidemia, diabetes mellitus, hypothyroidism and coronary artery disease. ${ }^{6,29-31}$ Hence, appropriate thromboprophylaxis especially in high-risk patients should not be neglected in cancer management. The current guidelines, however, do not recommend routine use of oral thromboprophylaxis in these patients, especially in ambulatory care settings, despite multiple thrombotic risk factors present due to the underlying malignant disease. ${ }^{7}$

Parenteral anticoagulants, such as LMWH and UFH, are the most commonly used agents as for thromboprophylaxis or VTE treatment in the inpatient care settings. ${ }^{7,32}$ The preference of these agents, nonetheless, has declined due to major bleeding risk and adherence issues related to the inconvenient route of administration; several studies have thus far evaluated the thromboprophylactic effects of oral anticoagulants, both in cancer and non-cancer patients, as an alternative option to replace 
parenteral agents. ${ }^{13-15,33,34}$ Two RCTs with the follow-up of 180 days demonstrated the efficacy of apixaban and rivaroxaban, respectively, along with an improved safety profile in terms of lower major bleeding risk in cancer patients as compared to placebo. ${ }^{13,14}$ In consistent with these results, our study confirmed that NOACs significantly reduced thrombosis risks in terms of VTE as a whole and more specifically in DVT among cancer patients; bleeding risk was also assessed lower in those receiving NOACs relative to non-users. NOACs (edoxaban, rivaroxaban and apixaban) led to significantly decreased VTE recurrences relative to dalteparin, a parenteral anticoagulant, in cancer patients, suggesting that NOACs can be a promising alternative to parenteral agents as thromboprophylaxis in cancer patients. ${ }^{34}$ Additionally, NOACs have similar properties to LMWH in terms of immediate onset of action and short halflives, ${ }^{35}$ and the oral route is more convenient for cancer patients.

In real-world practice, warfarin is not recommended as antithrombotic prophylaxis, particularly in cancer patients. Maintaining the International Normalized Ratio (INR) within the therapeutic range can be challenging in cancer patients, because of advanced age, frailty, drug interactions with various cancer-directed therapies, chemotherapyinduced adverse effects and cancer-related complications and comorbidities. ${ }^{32,36}$ According to Rose et al, cancer patients stayed in the target INR range for only 54\% of the time; their INR values were more variable and they experienced more thrombotic events than those without cancer. ${ }^{36}$ On the other hand, NOACs, which directly inhibit either thrombin (dabigatran) or direct factor Xa (rivaroxaban, apixaban, and edoxaban), do not require INR monitoring and frequent dose adjustments during the treatment. The present study showed no beneficial effects in the prevention of thrombosis with warfarin use relative to no-use. However, the head-to-head analysis revealed comparable effects between NOACs and warfarin, albeit potentially underpowered; the relatively small sample size in the warfarin cohort and its matched comparison group might have contributed to these results. We can also assume that warfarin may have some downfalls that need to be evaluated; according to the current treatment guidelines, warfarin is not recommended for acute VTE treatment in cancer patients because long-term anticoagulation with warfarin increased the incidence of recurrent VTE in cancer patients. ${ }^{32,37}$ Another study reported that warfarin demonstrated no benefits of thromboprophylaxis for cancer patients compared to parenteral anticoagulants due to high incidences of both recurrent VTE and adverse bleeding. $^{34}$

Our study examined differential effects of oral anticoagulation in accordance with patient gender, high-risk chemotherapy exposure, and Khorana index among cancer patients. Female sex is a well-known risk factor for VTE, but Roach et al suggested that a majority of VTE cases in female patients are associated with pregnancy and exogenous hormonal therapy, and males rather have higher risks of first and recurrent VTE than females when disregarding reproductive risk factors. ${ }^{1,38}$ In the present study, the incidence rates of VTE, regardless of its type of DVT or PE, were higher among female patients than in male patients in all comparison pairs. NOACs substantially decreased the risk of VTE, primarily DVT risk, in both sexes compared to no-use. On the other hand, bleeding risk was substantially lower in male cancer patients on NOACs, when compared to no-use, while such effects were not seen among female cancer patients. Interestingly, in the headto-head comparison between NOACs and warfarin, NOAC-receiving patients who had been exposed to highrisk chemotherapy were at a lower risk of DVT than those on warfarin. There are not enough studies evaluating the differential efficacy and safety of thromboprophylaxis by gender in cancer patients to confirm the validity of our study results. However, based on the studies assessing the clinical outcomes of cancer patients with VTE, female patients may show better outcomes than male patients during anticoagulation treatment composed of parenteral or oral agents, in terms of lower risk of fatal bleeding, death, and recurrent thrombosis after discontinuation of anticoagulant therapy. ${ }^{39,40}$ More studies evaluating gender differences in the thromboprophylactic efficacy and safety of oral anticoagulants among cancer patients are needed to provide optimal thromboprophylaxis in consideration of patient-specific factors and differential risk assessment.

\section{Limitations}

This study has several limitations. First, due to this study being a retrospective cohort study using national health insurance data, incorrect or missing documentation of diagnostic codes in claims data could have influenced the distribution of comorbidities at baseline as well as study outcomes. Laboratory data were not available from the HIRA database, which potentially led to underestimation of Khorana scores in individual study patients. Due to heterogeneity between oral anticoagulant users and non- 
users in real-world data, some of the baseline comorbidities and concomitant medications (antiplatelets, NSAIDs, and corticosteroids) were not fully balanced with PS matching, and had to be additionally adjusted for by the multiple logistic regression analysis in order to minimize potential confounding effects. Although NOACs have been associated with increased risk of gastrointestinal, gynecological and urological bleeding in other studies, ${ }^{41,42}$ such risk was not detected in the present study, which might have been affected by the aforementioned deferential comedication patterns between groups. Due to the limited number of NOAC-using cancer patients satisfying the inclusion criteria for study entry, we had to collectively categorize all the patients on any of the NOAC agents as the NOAC group, regardless of prophylactic or treatment doses used. Despite these limitations, the findings of the present study reveal the clinically significant benefits of NOAC use in cancer patients who have Khorana score of 1 or above. Further studies with more clinically relevant data including laboratory results and a larger sample size representing each of the NOAC agents are warranted to guide risk-based personalized, risk-based approach in thromboprophylaxis in cancer patients.

\section{Conclusions}

NOACs showed substantial effects in preventing not only VTE complications, primarily DVT events, but also adverse bleeding events in both male and female cancer patients as well as those with a Khorana score $\geq 1$, as compared to no-use. In contrast, no protective effects were found with warfarin compared to no-use in controlling the risk of thrombosis and adverse bleeding. DVT risk in those cancer patients exposed to high-risk chemotherapy was significantly lower with NOACs than with warfarin. NOACs can be an effective and safe thromboprophylactic option in both male and female cancer patients with increased risk for thrombosis complications.

\section{PI Statement}

The authors confirm that the Principal Investigators for this paper are Sooyoung Shin and Hwi-yeol Yun.

\section{Acknowledgments}

Yeo Jin Choi, Yong Won Choi and Jung-woo Chae are first authors. This study was supported by Basic Science Research Program through the National Research
Foundation of Korea (NRF) grant funded by the Ministry of Science and ICT (No. 2021R1C1C1003735) and by Ajou University research fund (S-2020-G0001-00381).

\section{Disclosure}

The authors report no conflicts of interest in this research.

\section{References}

1. Anderson FA, Spencer FA. Risk factors for venous thromboembolism. Circulation. 2003;107(Suppl 90231):I9-16. doi:10.1161/01.CIR.0000078469.07362.E6

2. Khorana AA, Connolly GC. Assessing risk of venous thromboembolism in the patient with cancer. $J$ Clin Oncol. 2009;27 (29):4839-4847. doi:10.1200/JCO.2009.22.3271

3. Blom JW, Doggen CJ, Osanto S, Rosendaal FR. Malignancies, prothrombotic mutations, and the risk of venous thrombosis. JAMA. 2005;293(6):715-722. doi:10.1001/jama.293.6.715

4. Campello E, Henderson MW, Noubouossie DF, Simioni P, Key NS. Contact system activation and cancer: new insights in the pathophysiology of cancer-associated thrombosis. Thromb Haemost. 2018;118 (2):251-265. doi:10.1160/TH17-08-0596

5. Connolly GC, Francis CW. Cancer-associated thrombosis. Hematology Am Soc Hematol Educ Program. 2013;2013:684-691. doi:10.1182/asheducation-2013.1.684

6. Abdol Razak NB, Jones G, Bhandari M, Berndt MC, Metharom P. Cancer-associated thrombosis: an overview of mechanisms, risk factors, and treatment. Cancers (Basel). 2018;10(10):380. doi:10.3390/ cancers 10100380

7. Key NS, Khorana AA, Kuderer NM, et al. Venous thromboembolism prophylaxis and treatment in patients with cancer: ASCO clinical practice guideline update. $J$ Clin Oncol. 2020;38(5):496-520. doi:10.1200/JCO.19.01461

8. Ihadaddene R, Carrier M. Thromboprophylaxis for medical patients with cancer: what do the guidelines say? Clin Pract. 2014;11 (2):155-168. doi:10.2217/cpr.14.2

9. Lyman GH, Bohlke K, Khorana AA, et al.; American Society of Clinical Oncology. Venous thromboembolism prophylaxis and treatment in patients with cancer: American society of clinical oncology clinical practice guideline update 2014. J Clin Oncol. 2015;33 (6):654-656. doi:10.1200/JCO.2014.59.7351

10. Angelini DE, Radivoyevitch T, McCrae KR, Khorana AA. Bleeding incidence and risk factors among cancer patients treated with anticoagulation. Am J Hematol. 2019;94(7):780-785. doi:10.1002/ ajh. 25494

11. Al-Samkari H, Connors JM. Managing the competing risks of thrombosis, bleeding, and anticoagulation in patients with malignancy. Blood Adv. 2019;3(22):3770-3779. doi:10.1182/ bloodadvances.2019000369

12. Shin S, Noh Y. Increased risk of adverse drug events secondary to bevacizumab treatment in patients with advanced or metastatic breast cancer: a meta-analysis of randomized controlled trials. Ther Clin Risk Manag. 2018;14:833-847. doi:10.2147/TCRM.S148840

13. Carrier M, Abou-Nassar K, Mallick R, et al.; AVERT Investigators. Apixaban to prevent venous thromboembolism in patients with cancer. $N$ Engl J Med. 2019;380(8):711-719. doi:10.1056/ NEJMoa 1814468

14. Khorana AA, Soff GA, Kakkar AK, et al.; CASSINI Investigators. Rivaroxaban for thromboprophylaxis in high-risk ambulatory patients with cancer. $N$ Engl J Med. 2019;380(8):720-728. doi:10.1056/ NEJMoa 1814630

15. Harter K, Levine M, Henderson SO. Anticoagulation drug therapy: a review. West J Emerg Med. 2015;16(1):11-17. doi:10.5811/ westjem.2014.12.22933 
16. Khorana AA, Kuderer NM, Culakova E, Lyman GH, Francis CW. Development and validation of a predictive model for chemotherapy-associated thrombosis. Blood. 2008;111 (10):4902-4907. doi:10.1182/blood-2007-10-116327

17. Debbie Jiang MD, Alfred Ian Lee MD. Thrombotic risk from chemotherapy and other cancer therapies. Cancer Treat Res. 2019;179:87-101. doi:10.1007/978-3-030-20315-3_6

18. Choi YJ, Kim DJ, Shin S. Incident cancer risk in dipeptidyl peptidase-4 inhibitor-treated patients with type 2 diabetes mellitus. Cancer Manag Res. 2019;11:7427-7438. doi:10.2147/CMAR. S215107

19. Mulder FI, Candeloro M, Kamphuisen PW, et al.; CAT-prediction collaborators. The Khorana score for prediction of venous thromboembolism in cancer patients: a systematic review and meta-analysis. Haematologica. 2019;104(6):1277-1287. doi:10.3324/haematol.2018.209114

20. Agnelli G, Verso M. Management of venous thromboembolism in patients with cancer. J Thromb Haemost. 2011;9(Suppl 1):316-324. doi:10.1111/j.1538-7836.2011.04346.x

21. Blom JW, Vanderschoot JP, Oostindiër MJ, Osanto S, van der Meer FJ, Rosendaal FR. Incidence of venous thrombosis in a large cohort of 66,329 cancer patients: results of a record linkage study. $J$ Thromb Haemost. 2006;4(3):529-535. doi:10.1111/j.15387836.2006.01804.x

22. Rak J, Milsom C, Magnus N, Yu J. Tissue factor in tumour progression. Best Pract Res Clin Haematol. 2009;22(1):71-83. doi:10.1016/j.beha.2008.12.008

23. Lima LG, Monteiro RQ. Activation of blood coagulation in cancer: implications for tumour progression. Biosci Rep. 2013;33(5):e00064 doi:10.1042/BSR20130057

24. Han X, Guo B, Li Y, Zhu B. Tissue factor in tumor microenvironment: a systematic review. J Hematol Oncol. 2014;7:54. doi:10.1186/ s13045-014-0054-8

25. Smith AW, Reeve BB, Bellizzi KM, et al. Cancer, comorbidities, and health-related quality of life of older adults. Health Care Financ Rev. 2008;29(4):41-56.

26. Khorana AA, Dalal M, Lin J, Connolly GC. Incidence and predictors of venous thromboembolism (VTE) among ambulatory high-risk cancer patients undergoing chemotherapy in the United States. Cancer. 2013;119(3):648-655. doi:10.1002/cncr.27772

27. Silverstein MD, Heit JA, Mohr DN, Petterson TM, O'Fallon WM, Melton LJ. Trends in the incidence of deep vein thrombosis and pulmonary embolism: a 25-year population-based study. Arch Intern Med. 1998;158(6):585-593. doi:10.1001/archinte.158.6.585

28. White MC, Holman DM, Boehm JE, Peipins LA, Grossman M, Henley SJ. Age and cancer risk: a potentially modifiable relationship. Am J Prev Med. 2014;46(Suppl 3):S7-15. doi:10.1016/ j.amepre.2013.10.029

29. Roy S, Vallepu S, Barrios C, Hunter K. Comparison of comorbid conditions between cancer survivors and age-matched patients without cancer. J Clin Med Res. 2018;10(12):911-919. doi:10.14740/ jocmr3617w
30. Khorana AA, Francis CW, Culakova E, Kuderer NM, Lyman GH. Frequency, risk factors, and trends for venous thromboembolism among hospitalized cancer patients. Cancer. 2007;110 (10):2339-2346. doi:10.1002/cncr.23062

31. Noh Y, Jeon SM, Shin S. Association between glucose-lowering treatment and cancer metastasis among patients with preexisting type 2 diabetes and incident malignancy. Int $J$ Cancer. 2019;144 (7):1530-1539. doi:10.1002/ijc.31870

32. Iorga RA, Bratu OG, Marcu RD, et al. Venous thromboembolism in cancer patients: still looking for answers. Exp Ther Med. 2019;18 (6):5026-5032. doi:10.3892/etm.2019.8019

33. Aikens GB, Osmundson JR, Rivey MP. New oral pharmacotherapeutic agents for venous thromboprophylaxis after total hip arthroplasty. World J Orthop. 2014;5(3):188-203. doi:10.5312/wjo.v5.13.188

34. Song AB, Rosovsky RP, Connors JM, Al-Samkari H. Direct oral anticoagulants for treatment and prevention of venous thromboembolism in cancer patients. Vasc Health Risk Manag. 2019;15:175-186. doi:10.2147/VHRM.S132556

35. Al-Samkari H, Connors JM. The role of direct oral anticoagulants in treatment of cancer-associated thrombosis. Cancers (Basel). 2018;10 (8):271. doi:10.3390/cancers 10080271

36. Rose AJ, Sharman JP, Ozonoff A, Henault LE, Hylek EM. Effectiveness of warfarin among patients with cancer. J Gen Intern Med. 2007;22(7):997-1002. doi:10.1007/s11606-007-0228-y

37. Lee AY, Levine MN. Venous thromboembolism and cancer: risks and outcomes. Circulation. 2003;107(Suppl 90231):I17-21. doi:10.1161/ 01.CIR.0000078466.72504.AC

38. Roach RE, Cannegieter SC, Lijfering WM. Differential risks in men and women for first and recurrent venous thrombosis: the role of genes and environment. J Thromb Haemost. 2014;12(10):1593-1600. doi:10.1111/jth. 12678

39. Martín-Martos F, Trujillo-Santos J, Barrón M, et al.; RIETE Investigators. Gender differences in cancer patients with acute venous thromboembolism. Thromb Res. 2015;135(Suppl 1):S12-5. doi:10.1016/S0049-3848(15)50433-7

40. McRae S, Tran H, Schulman S, Ginsberg J, Kearon C. Effect of patient's sex on risk of recurrent venous thromboembolism: a metaanalysis. Lancet. 2006;368(9533):371-378. doi:10.1016/S01406736(06)69110-1

41. Clemens A, Strack A, Noack H, Konstantinides S, Brueckmann M, Lip GY. Anticoagulant-related gastrointestinal bleeding-could this facilitate early detection of benign or malignant gastrointestinal lesions? Ann Med. 2014;46(8):672-678. doi:10.3109/ 07853890.2014 .952327

42. Campello E, Spiezia L, Simion C, et al. Direct oral anticoagulants in patients with inherited thrombophilia and venous thromboembolism: a prospective cohort study. J Am Heart Assoc. 2020;9(23):e018917. doi:10.1161/JAHA.120.018917
Risk Management and Healthcare Policy

\section{Publish your work in this journal}

Risk Management and Healthcare Policy is an international, peerreviewed, open access journal focusing on all aspects of public health, policy, and preventative measures to promote good health and improve morbidity and mortality in the population. The journal welcomes submitted papers covering original research, basic science, clinical \& epidemiological studies, reviews and evaluations, guidelines, expert opinion and commentary, case reports and extended reports. The manuscript management system is completely online and includes a very quick and fair peer-review system, which is all easy to use. Visit http://www.dovepress.com/testimonials.php to read real quotes from published authors. 